Sepsis/Infection

\title{
Early procalcitonin kinetics and appropriateness of empirical antimicrobial therapy in critically ill patients ${ }^{\text {tr }}$ A prospective observational study
}

\author{
Domonkos Trásy, MD ${ }^{\mathrm{a}, *}$, Krisztián Tánczos, MD a , Márton Németh, MD a , Péter Hankovszky, MD a , \\ András Lovas, MD ${ }^{\mathrm{a}}$, András Mikor, MD ${ }^{\mathrm{a}}$, Ildikó László, MD ${ }^{\mathrm{a}}$, Edit Hajdú, MD ${ }^{\mathrm{b}}$, Angelika Osztroluczki ${ }^{\mathrm{a}}$, \\ János Fazakas, $\mathrm{MD}^{\mathrm{c}}$, Zsolt Molnár, MD ${ }^{\mathrm{a}}$ The EProK study group \\ a University of Szeged, Faculty of Medicine, Department of Anaesthesiology and Intensive Therapy, Szeged, Hungary \\ b University of Szeged, Faculty of Medicine, Division of Infectious Diseases, First Department of Internal Medicine, Szeged, Hungary \\ c Semmelweis University, Faculty of Medicine, Department of Transplantation and Surgery, Budapest, Hungary
}

\section{A R T I C L E I N F O}

\section{Keywords:}

Infection

Empirical antimicrobial therapy

Appropriate antimicrobial therapy

Procalcitonin

Biomarkers

Sepsis

\begin{abstract}
A B S T R A C T
Purpose: The purpose was to investigate the value of procalcitonin (PCT) kinetics in predicting the appropriateness of empirical antimicrobial treatment in critically ill patients.

Materials and methods: This prospective observational study recruited patients in whom empirical antimicrobial therapy was started for suspected infection. Biochemical and physiological parameters were measured before initiating antimicrobials $\left(t_{0}\right), 8$ hourly $\left(t_{8}, t_{16}, t_{24}\right)$, and then daily ( day $\left._{2-6}\right)$. Patients were grouped post hoc into appropriate (A) and inappropriate (IA) groups.

Results: Of 209 patients, infection was confirmed in 67\%. Procalcitonin kinetics were different between the IA $(\mathrm{n}=33)$ and A groups $(\mathrm{n}=108)$. In the IA group, PCT levels (median [interquartile range]) increased: $t_{0}=$ 2.8 (1.2-7.4), $t_{16}=8.6$ (4.8-22.1), $t_{24}=14.5$ (4.9-36.1), $P<.05$. In the A group, PCT peaked at $t_{16}$ and started to decrease by $t_{24}: t_{0}=4.2(1.9-12.8), t_{16}=6.99$ (3.4-29.1), $t_{24}=5.2(2.0-16.7), P<.05$. Receiver operating characteristic analysis revealed that a PCT elevation greater than or equal to $69 \%$ from $t_{0}$ to $t_{16}$ had an area under the curve for predicting inappropriate antimicrobial treatment of 0.73 (95\% confidence interval, $0.63-0.83$ ), $P<.001 ;$ from $t_{0}$ to $t_{24}$, a greater than or equal to $74 \%$ increase had an area under the curve of $0.86(0.77-0.94), P<.001$. Hospital mortality was $37 \%$ in the A group and $61 \%$ in the IA group $(P=.017)$.

Conclusions: Early response of PCT in the first 24 hours of commencing empirical antimicrobials in critically ill patients may help the clinician to evaluate the appropriateness of therapy.
\end{abstract}

(C) 2016 Elsevier Inc. All rights reserved.

\section{Introduction}

Sepsis remains the leading cause of death among critically ill patients worldwide [1,2]. It is well documented that delaying appropriate antimicrobial treatment increases mortality [3,4], but empirical antimicrobials have been proven to be inadequate in almost $30 \%$ of cases [5]. Diagnosing infection and assessing the progress of the

\footnotetext{
is Trial registration: ClinicalTrials.gov identifier: NCT02294695.

* Corresponding author at: Department of Anaesthesiology and Intensive Therapy, University of Szeged, 6. Semmelweis str., 6725 Szeged, Hungary.

E-mail addresses: trasydom@gmail.com (D. Trásy), tkrisztian78@gmail.com (K. Tánczos), nemethmarton85@gmail.com (M. Németh), hankovszky@gmail.com (P. Hankovszky), anlovas@gmail.com (A. Lovas), andrasmikor@gmail.com (A. Mikor), ildiko.laszlo88@gmail.com (I. László), horvathne.hajdu.edit@med.u-szeged.hu (E. Hajdú), oszang78@gmail.com (A. Osztroluczki), jancsidora@gmail.com (J. Fazakas), zsoltmolna@gmail.com (Z. Molnár).
}

patients' condition have been supported by biomarkers for decades. Procalcitonin (PCT) and C-reactive protein (CRP) are the most commonly used biomarkers in the clinical setting, of which PCT seems to have a better sensitivity and specificity for differentiating bacterial infection from nonbacterial systemic inflammatory response [6-9]. There is considerable evidence that PCT-guided antimicrobial management considerably reduces antimicrobial use in lower respiratory tract infections, and it may also shorten the duration of antimicrobial treatment in the intensive care unit (ICU) [10,11].

However, during the initial phase of treatment, physicians often have no way of confirming the adequacy of the commenced antimicrobials. As PCT is a fast-reacting biomarker with a half-life of 24 hours, theoretically, it is possible that the early kinetics of PCT, within this first 24 hours after commencing empirical antimicrobial therapy, may reflect the efficacy of the treatment. Therefore, our aim was to perform a prospective observational study to investigate the value of PCT kinetics 
measured 8 hourly during the first 24 hours for predicting the appropriateness of empirical antimicrobial treatment in critically ill patients.

\section{Methods}

\subsection{Patient selection}

This prospective observational study was undertaken between October 2012 and October 2013 and was approved by the Regional and Institutional Human Medical Biological Research Ethics Committee, University of Szeged, Hungary (WHO-3005; 19.04.2012, Chairperson Prof T Wittmann). The investigation was performed at the University of Szeged (Szeged, Hungary) Albert Szent-Györgyi Health Center in a 27-bed multidisciplinary tertiary ICU. The study was registered at ClinicalTrials.gov with registration number NCT02294695. Written informed consent was obtained from all subjects or from their relatives.

\subsubsection{Inclusion criteria}

All patients older than 18 years with suspected infection on admission or during their stay on the ICU were screened for eligibility. Patients were enrolled when the attending physician suspected infection and empirical antimicrobial therapy was started.

\subsubsection{Exclusion criteria}

The exclusion criteria were as follows: age less than 18 years, antimicrobial therapy within 48 hours, conditions that have been shown to interfere with the inflammatory response such as acute renal replacement therapy in the first 24 hours [12] and cardiopulmonary resuscitation [13], patients with end-stage diseases, and immunocompromised patients.

\subsection{Subgroups and definitions}

Diagnosis of infection and appropriateness of the empirical antimicrobials were established based on recommendations [14], clinical parameters, and biochemical and microbiological results evaluated by 2 experts blinded for the PCT data apart from the first PCT result: an infectologist (EH) and an intensivist (JF). Patients were then grouped into infectious and noninfectious groups. Patients with suspected infection but negative microbiology were also excluded from the final analysis.

Antimicrobial therapy was evaluated by 2 independent experts ( $\mathrm{EH}$ and JF), and it was considered appropriate if ( $a$ ) the isolated pathogens were susceptible to at least 1 of the commenced antimicrobials [15] and (b) the appropriate dosage, as recommended by our local protocols, was administered. Based on these results, patients were grouped post hoc into appropriate (A group) and inappropriate (IA group) antimicrobial treatment groups.

Patients were further divided into "medical" and "surgical" groups. The medical group represented patients without surgical intervention. In the surgical group, infection either was related to surgery or required surgery for source control [16]. These groups were also further divided

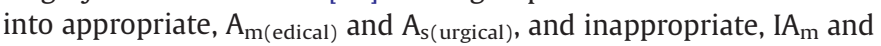
$\mathrm{IA}_{\mathrm{s}}$, groups.

\subsection{Protocol and data collection}

When infection was suspected (based on temperature, white blood cell count, clinical picture, PCT levels) by the attending physician, specimens were sent for microbiology, and antimicrobial therapy was commenced. The choice of antimicrobials was determined by local protocols based on international guidelines [17-19].

\subsubsection{Data collection}

After enrollment, demographic data, parameters of vital organ functions, and laboratory data were collected for 6 days. Length of ICU and hospital stay and mortality were also documented.

\subsubsection{PCT measurement}

Procalcitonin levels were determined immediately before the initiation of antimicrobials $\left(t_{0}\right), 8$ hourly $\left(t_{8}, t_{16}, t_{24}\right)$ during the first 24 hours, and then daily $\left(\mathrm{day}_{2}-\mathrm{day}_{6}\right)$. The flowchart of the data collection is summarized in Fig. 1.

Serum PCT levels were measured with Cobas 6000 analyzer (Hitachi High-Technologies Corporation, Tokyo, Japan). Analyzer reagents (Elecsys $\mathrm{B} \cdot \mathrm{R} \cdot \mathrm{A} \cdot \mathrm{H} \cdot \mathrm{M} \cdot \mathrm{S}$ PCT assay) were developed in collaboration with B $\cdot \mathrm{R} \cdot \mathrm{A} \cdot \mathrm{H} \cdot \mathrm{M} \cdot \mathrm{S}$ Corporation (Hennigsdorf, Germany) and Roche Diagnostics (Mannheim, Germany). Procalcitonin was determined by electrochemiluminescence immunoassay serum on the automated Roche Elecsys and Cobas immunoassay analyzers.

\subsubsection{Microbiological staining and antibiograms}

Microbiological tests were performed and sent at $t_{0}$ (before the first antimicrobial dose was administered) and, if necessary, repeated on the following days to identify microorganisms and their resistance. The type of antimicrobials, the dosage, the bacterial strains, and their antibiogram profile were recorded.

\subsection{Statistical analysis}

The primary end point of the study was the difference in PCT kinetics after 24 hours of starting the antimicrobial treatment. According to our former pilot study [20], a PCT increase of less than $70 \%$ within the first 24 hours compared with the baseline value $\left(t_{0}\right)$ had an $84 \%$ positive predictive value with $80 \%$ sensitivity and $41 \%$ specificity $(P=.059)$, indicating appropriate antimicrobial treatment. Therefore, for the study to have $80 \%$ power to show the smallest clinically relevant difference of $15 \%$, an increase of PCT between the A and IA groups (ie, 70\% increase in the IA group and 55\% increase of PCT in the A group from $t_{0}$ to $t_{24}$ ) with a $P<.05$, the required sample size was at least 161 patients. Based on this calculation, we decided to enroll patients for at least 12 months.

Data were analyzed using IBM SPSS Statistics Version 20 (Armonk, NY) and Systat Software Inc SigmaPlot 12.5 (London, UK) software. For continuous data, the Shapiro-Wilk tests were performed to assess normal distribution. Demographic data were analyzed between groups with the Student $t$ test or nonparametric data with the Mann-Whitney $U$ test as appropriate. Biomarkers were analyzed by using 2-way repeated-measures analysis of variances (all pairwise multiple comparison procedures: Holm-Sidak method). Categorical data were compared using $\chi^{2}$ tests. Receiver operating characteristic (ROC) curve and the respective areas under the curves (AUCs) were calculated for PCT and CRP levels. The best cutoff values were determined to maximize the Youden index $(J=\max [$ Sens + Spec -1$])$. The test parameters (sensitivity, specificity, and positive and negative predictive values) were compared by their $95 \%$ confidence intervals (CIs). A level of $P<.05$ was defined as statistically significant. Data are given as mean \pm standard deviation or median (25\%-75\% interquartile range) as appropriate.

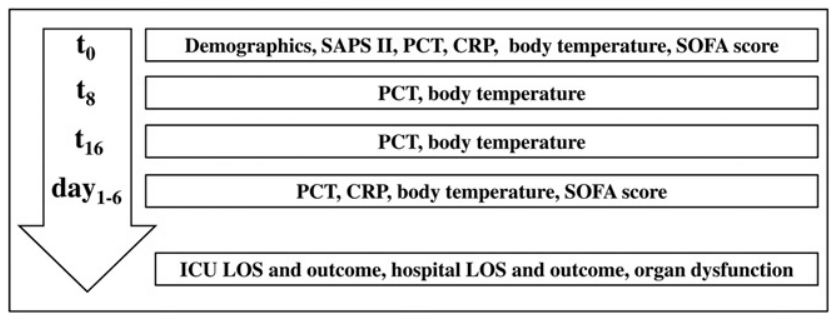

Fig. 1. Flowchart. $t_{0-24}$ indicates sampling within the first 24 hours after commencement of empirical antimicrobials; SAPS II, Simplified Acute Physiology Score II; SOFA, Sequential Organ Failure Assessment; LOS, length of stay. 
Table 1

Demographics and infection sources in the entire cohort

\begin{tabular}{|c|c|c|c|c|c|}
\hline & & Total $(\mathrm{N}=209)$ & Appropriate $\mathrm{AB}(\mathrm{n}=108)$ & Inappropriate $\mathrm{AB}(\mathrm{n}=33)$ & $P$ value \\
\hline \multicolumn{2}{|l|}{ Age $(y)$} & $68(19)$ & $68(19)$ & $69(20)$ & .842 \\
\hline \multicolumn{2}{|l|}{ Male, $\mathrm{n}(\%)$} & $117(56)$ & $60(55)$ & $16(48)$ & .476 \\
\hline \multicolumn{2}{|l|}{ Body height $(\mathrm{cm})$} & $170(12)$ & $169(14)$ & $165(19)$ & .422 \\
\hline \multicolumn{2}{|l|}{ Body weight (kg) } & $76(25)$ & $70(18)$ & $80(27)$ & .218 \\
\hline \multicolumn{2}{|l|}{ SAPS II points } & $67 \pm 19$ & $68 \pm 19$ & $72 \pm 16$ & .333 \\
\hline \multicolumn{2}{|l|}{ SAPS II PM } & $78(47)$ & $81(32)$ & $88(27)$ & .298 \\
\hline \multicolumn{2}{|c|}{ SOFA score points at $t_{0}$} & $14(5)$ & $14(4)$ & $15(6)$ & .298 \\
\hline \multicolumn{2}{|c|}{ delta SOFA score points $\left(t_{0}-t_{24}\right)$} & $0(2)$ & $0(2)$ & $0(2)$ & .568 \\
\hline \multicolumn{2}{|c|}{ ICU days before enrollment } & $1(2)$ & $0(2)$ & $1(2)$ & .798 \\
\hline \multicolumn{2}{|l|}{ ICU LOS $(\mathrm{d})$} & $8(9)$ & $8(10)$ & $9(11)$ & .263 \\
\hline \multicolumn{2}{|l|}{ ICU survival, n (\%) } & $151(72)$ & $84(78)$ & $14(42)$ & $<.001$ \\
\hline \multicolumn{2}{|l|}{ Hospital LOS (d) } & $15(17)$ & $16(22)$ & $17(16)$ & .444 \\
\hline \multicolumn{2}{|l|}{ Hospital survival (\%) } & $126(60)$ & $68(63)$ & $13(39)$ & .017 \\
\hline \multicolumn{2}{|l|}{ Mechanical ventilation (d) } & $4(8)$ & $4(9)$ & $7(8)$ & .011 \\
\hline \multicolumn{2}{|c|}{ Vasopressor therapy (d) } & $3(4)$ & $3(3)$ & $5(4)$ & .004 \\
\hline \multicolumn{2}{|c|}{ Renal replacement therapy, n (\%) } & $65(31)$ & $33(31)$ & $19(57)$ & .005 \\
\hline \multicolumn{2}{|c|}{ Nosocomial infection, $\mathrm{n}(\%)$} & & $53(49)$ & $16(48)$ & .953 \\
\hline \multirow[t]{7}{*}{ Source of infection, $\mathrm{n}(\%)$} & Respiratory & & $54(50)$ & $25(76)$ & .007 \\
\hline & Abdominal & & $19(18)$ & $5(15)$ & .744 \\
\hline & Soft tissue & & $15(14)$ & $2(6)$ & .227 \\
\hline & UTI & & $10(9)$ & $2(6)$ & .564 \\
\hline & BSI & & $7(6)$ & 0 & .134 \\
\hline & Meningitis & & $5(5)$ & 0 & .208 \\
\hline & Other & & $1(1)$ & $1(3)$ & .371 \\
\hline
\end{tabular}

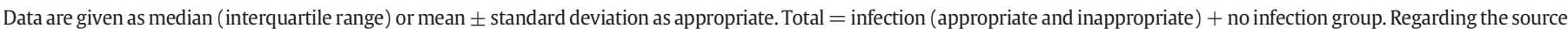

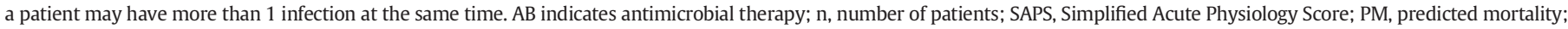
LOS, length of stay; UTI, urinary tract infection; BSI, bloodstream infection.

\section{Results}

Over the study period, 209 patients were enrolled. Demographics are summarized in Table 1. From the 209 patients, 141 (67\%) had proven infection, with infection unproven in 44 (21\%). In 24 patients, although infection was highly likely, microbiology did not reveal pathogens; hence, these subjects were excluded from the final analysis. Procalcitonin at $t_{0}$ was significantly higher in the infectious group compared with noninfectious group: 4.53 (1.76-16.30) vs $1.0(0.13-2.98) \mathrm{ng} / \mathrm{mL}, P=.024$, respectively. In the infectious group $(\mathrm{n}=141), 108(77 \%)$ patients received appropriate antimicrobial therapy (A group), and in 33 (23\%), antimicrobials proved to be inappropriate (IA group). Detailed descriptions of the source and pathogens are summarized in Tables S4 and S5.

Regarding demographics, there were no differences between the $\mathrm{A}$ and IA groups, but ICU and hospital survival was significantly higher in the A group (Table 1). These patients also required less vasopressors and renal replacement therapy compared with the IA group. From the 33 patients in the IA group, antimicrobials were changed in 20 patients on day 2 or 3, without any significant effect on PCT kinetics, compared with the other 18 patients (data not shown).

\subsection{PCT kinetics}

In both groups, the increase in PCT levels continued after the initiation of empirical antimicrobial treatment $\left(t_{0}\right)$ until 16 hours $\left(t_{16}\right)$ (Fig. 2A). In the IA group, there was a significant increase from $t_{16}$ to $t_{24}$, whereas in the A group, there was a significant decrease from $t_{16}$ to $t_{24}$. By $t_{24}$, the PCT reached significantly higher levels in the IA group and remained higher the following day compared with the A group. In the A group, PCT levels peaked at $t_{16}$, whereas in the IA group, the peak was at $t_{24}$. From $t_{24}$ until the fifth day, PCT levels decreased in both groups (Fig. 2A).

There was a nonsignificant increase in CRP from $t_{0}$ to $t_{24}$ in both groups. In the A group, CRP peaked at $t_{24}$. In the IA group, CRP remained high on $\mathrm{day}_{2}$, with levels remaining higher compared with the A group on days ${ }_{3-5}$. After day 2 , CRP levels decreased in both groups (Fig. S4A). Body temperature showed no significant change over time (Fig. S4B).
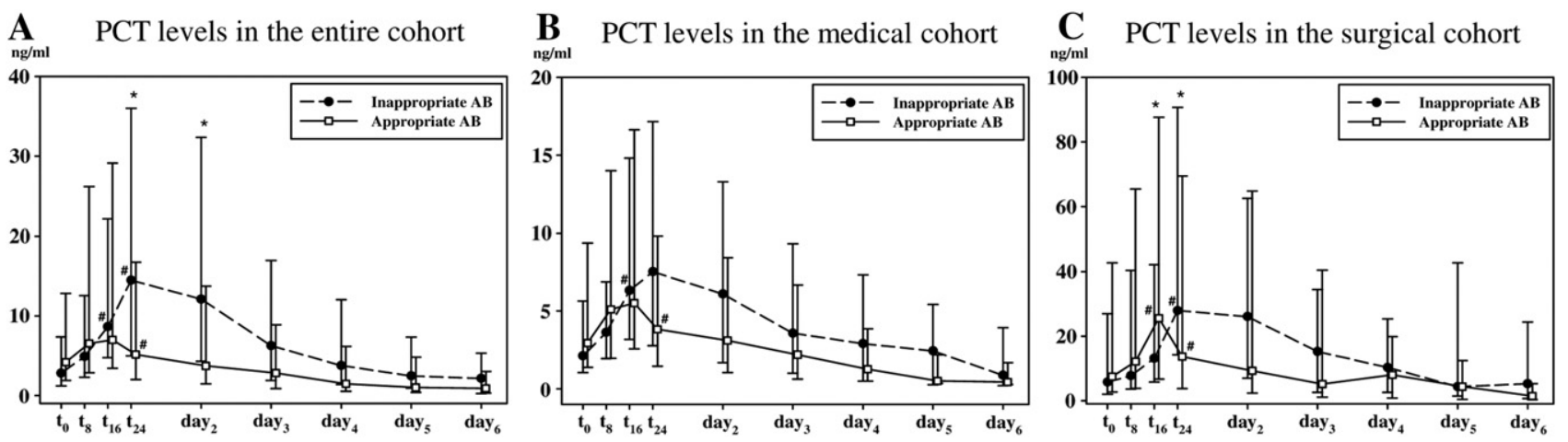

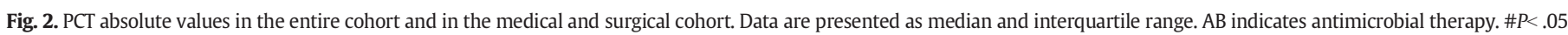
within groups; ${ }^{*} P<.05$ between groups. 


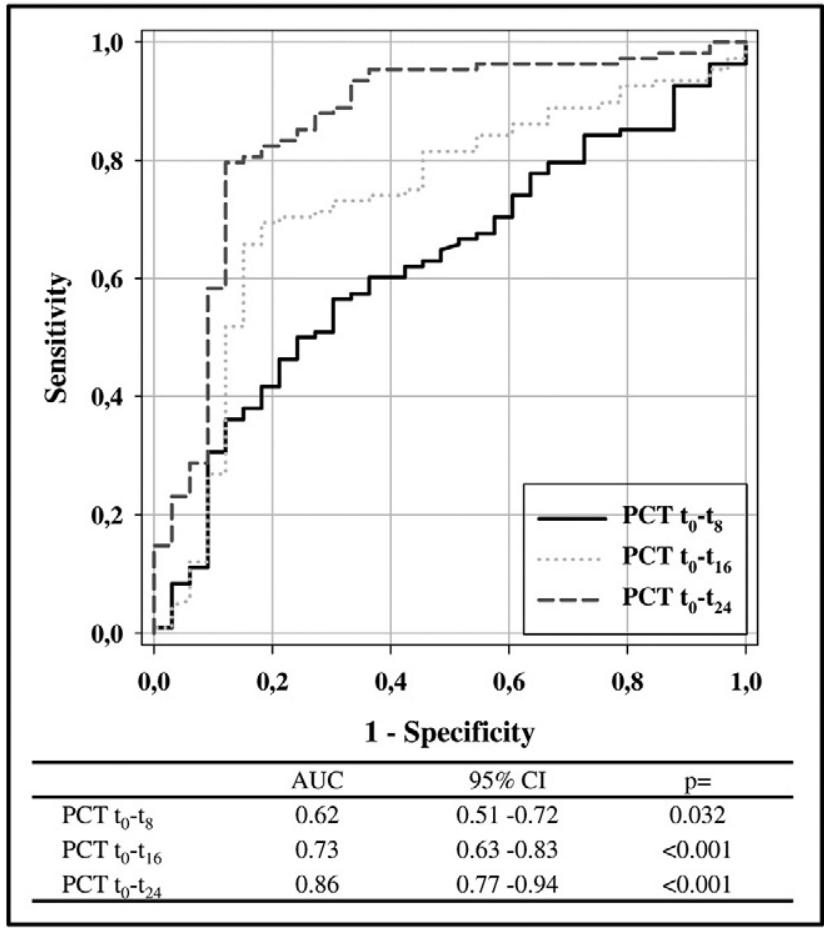

Fig. 3. Receiver operating characteristic curve.

\subsection{PCT kinetics in medical and surgical patients}

In patients with infection, $\mathrm{PCT}$ followed similar kinetics in medical $(\mathrm{n}=$ 91 ) and surgical $(n=50)$ patients, with substantial differences in the absolute values at $t_{0}$ (median: 2.74 [25\%-75\% interquartile range: $\left.1.30-7.72\right]$ vs 6.46 [2.61-40.07], $P=.002)$ and at $t_{24}(4.41$ [1.52-13.55] vs 17.02 [6.74$69.45] \mathrm{ng} / \mathrm{mL}, P<.001$, respectively). Medical and surgical patients were further divided into appropriate- $A_{m}(n=70)$ and $A_{s}(n=38)$-and inappropriate- $I A_{m}(n=21)$ and $I A_{s}(n=12)$-subgroups. Kinetics in all subgroups followed the same pattern as described for the whole sample (Fig. 2B and C).

Kinetics of CRP were similar in both groups, with no significant differences within and between groups during the study period (Fig. S5A and $\mathrm{B}$ ). The same holds true for body temperature, with the only difference being that, in the medical cohort at $t_{24}$, temperature was higher for the next 3 days (Fig. S5C and D) (Table S3).

\subsection{Predictive value of PCT for indicating appropriate antimicrobial treatment}

The ROC analysis revealed that a PCT elevation from $t_{0}$ to $t_{16}$ had an AUC of $0.73(95 \% \mathrm{CI}, 0.63-0.83 ; P<.001)$ and that from $t_{0}$ to $t_{24}$ had an AUC of 0.86 (95\% CI, 0.77-0.94; $P<.001$ ) (Fig. 3). According to the Youden index, the best cutoff for PCT increase from $t_{0}$ to $t_{16}$ was $69.2 \%$, and from $t_{0}$ to $t_{24}$, it was $73.5 \%$ (Table 2 ).

\section{Discussion}

The main findings of this study were that PCT kinetics during the first 24 hours after commencing empirical antimicrobial therapy show significant differences in patients on appropriate antimicrobial therapy compared with those on inappropriate antimicrobial therapy and that there were significantly higher absolute PCT values but similar kinetics in surgical compared with medical patients with infections.

\subsection{Diagnosing infection}

Although the classical definitions of sepsis syndromes[21] and consensus criteria of sepsis [22] have been implemented worldwide for decades, differentiating systemic inflammatory response from bacterial infection remains a challenge $[23,24]$, and mortality from sepsis and septic shock is still very high. This uncertainty in the diagnosis may lead to unnecessary overuse of antimicrobials resulting in increased bacterial resistance, adverse effects from the antimicrobials, and increased costs [25].

In this diagnostic dilemma, there has been considerable interest in the use of infection/sepsis biomarkers. Among these biomarkers, the two most commonly used are PCT and CRP. Several studies have shown that PCT has a better sensitivity and specificity for indicating bacterial infections than CRP [26]. This was confirmed by the results from this study, where only PCT was able to differentiate patients with proven infection from patients with no infection. These results are similar to those reported by recent large randomized trials $[10,11]$.

\subsection{Appropriate antimicrobial therapy}

Inappropriate empirical antimicrobials have a strong adverse effect on survival [27], although such therapy is a common feature in the ICU, reportedly as high as $25 \%$ to $30 \%$ [5,15]. Once inappropriate antimicrobials are initiated, it often takes days (until organism isolation and sensitivities are produced) to correct them. In our study, $23 \%$ of patients received inappropriate antimicrobials. This high incidence may be due to the lack of fast and reliable diagnostic tests for bacterial infections and to the subsequent delay in microbiological results. Earlier recognition of potential inappropriate microbial therapy may allow an opportunity to substantially improve outcome.

To our knowledge, this is the first study to show that the early kinetics of PCT measured within the first 24 hours may help clinicians to evaluate the appropriateness of empirical antimicrobial therapy in critically ill patients. The rationale for measuring successive PCT levels within this time frame came from the assumption that, by giving appropriate antibiotics, this may slow the inflammatory response within hours and that this could be detected by serial measurements of PCT.

In a study by Charles et al [15], they observed a signal of lower PCT levels on day 2 in the appropriate group, but the difference was not significant. This may be due to the fact that, in their study, PCT was measured retrospectively; therefore, the time elapsed between the PCT measurements and the antibiotic therapy was uncontrolled, unlike this study where investigating PCT kinetics as precisely as possible during the first 24 hours was a particular aim. In this study, although PCT continued to increase after the initiation of empirical antimicrobial

Table 2

Cutoff values for appropriate and inappropriate antimicrobial treatment in the entire cohort

\begin{tabular}{|c|c|c|c|c|c|c|}
\hline & Cutoff value & Sensitivity $(95 \% \mathrm{CI})$ & Specificity (95\% CI) & PPV (95\% CI) & NPV (95\% CI) & $P$ \\
\hline PCT $t_{0}-t_{8}$ & $\geq 45.6 \%$ & $56.5 \%(0.46-0.66)$ & $69.7 \%(0.51-0.84)$ & $31.7 \%(0.28-0.54)$ & $85.4 \%(0.78-0.92)$ & .090 \\
\hline PCT $t_{0}-t_{16}$ & $\geq 69.2 \%$ & $65.7 \%(0.56-0.74)$ & $84.8 \%(0.68-0.94)$ & $42.1 \%(0.33-0.60)$ & $92.8 \%(0.82-0.95)$ & .048 \\
\hline PCT $t_{0}-t_{24}$ & $\geq 73.5 \%$ & $79.6 \%(0.70-0.86)$ & $87.8 \%(0.71-0.96)$ & $53.5 \%(0.41-0.70)$ & $95.2 \%(0.84-0.98)$ & $<.001$ \\
\hline
\end{tabular}

The best cutoff value was determined using the Youden index. PPV indicates positive predictive value; NPV, negative predictive value. 
treatment during the first 16 hours in both the A and IA groups, in the A group, there was a significant PCT decrease during the next 8 hours $\left(t_{24}\right)$, whereas in the IA group, PCT continued to increase, reached a significantly higher level by $t_{24}$, and remained higher the next day compared with the A group. A PCT increase of at least 69.2\% during the first 16 hours or a PCT increase of at least 73.5\% during the first 24 hours was the best cutoff value to indicate inappropriate antimicrobial treatment. It is known that PCT increases within hours after an infectious insult and levels halve daily once the infection is under control [28]. This feature explains the significant difference found in the PCT kinetics between the A and IA groups during the first 24 hours. It is also important to note that, after day 1, PCT decreased in both groups, although PCT levels remained significantly higher in the IA group. This is in line with the results from Charles et al [15], who measured PCT during the first 4 days of treatment and found a decrease from day 2 to day 3 in both the appropriate and inappropriate groups, but the decrease was more pronounced in the appropriate group. This can be explained by the finding that adequate supportive therapy on its own may attenuate the inflammatory response [29]. Furthermore, monocytes become exhausted after a certain period of time, also affecting PCT production $[30,31]$. It may be of significance that the grouping was based on the initial antibiotic therapy; hence, no patients were "crossed over" from the inappropriate to the appropriate group. However, from the 33 patients in the IA group, antibiotics were changed on day 2 or day 3 in 18 cases. We analyzed and compared PCT kinetics in patients in whom we changed $(n=18)$ as compared with patients in whom we did not change ( $\mathrm{n}=15)$ antibiotics over the study period but found no significant differences (data not shown).

Translating the results of the current study into clinical practice means that, when measuring PCT on commencement of antimicrobials $\left(t_{0}\right)$ and then at 16 and 24 hours, a "large" increase within the first 16 to 24 hours ( $\geq 69.2 \%-73.5 \%)$ may indicate inappropriate antimicrobial therapy, whereas a lower-grade increase or a decreasing tendency after 16 hours would support appropriate antimicrobial therapy. The clinical importance of our findings is emphasized by the significant difference in hospital mortality between the A group and the IA group (37\% vs 61\%).

Another important finding of the current study is that CRP did not differentiate between the appropriate and inappropriate groups within the first 48 hours. This is in accordance with previously published data indicating that CRP is a "slow" marker and not as reliable as PCT in the critically ill $[32,33]$. The same holds true for body temperature, which, as with other studies, highlights that its use for guiding antimicrobial therapy is questionable [23].

\subsection{PCT kinetics in surgical as compared with medical patients}

Our results also support that PCT is several times higher in surgical compared with medical patients, but we also found that early kinetics were similar to those found in the whole sample. Our data also suggest that percentage changes of PCT may be a better, universally applicable approach for monitoring treatment progress rather than absolute values.

There is strong evidence that, because of direct cellular damage as in severe trauma, major surgery, and after ischemia-reperfusion, also known as damage-associated molecular patterns, there is an inflammatory mediator release very similar to that following an infectious insult, called pathogen-associated molecular patterns (PAMPs) [34]. Therefore, unspecific elevations in PCT levels can typically be seen in the absence of a bacterial infection $[33,35]$. Theoretically, in surgical patients with sepsis, damage-associated molecular pattern and PAMP take place at the same time, leading to a pronounced inflammatory response, whereas in medical patients, PAMP may occur on its own, resulting in a less extensive inflammatory response [16]. This feature is the reason why the same absolute values of PCT may mean completely different information in a medical compared with a surgical patient but, as shown in our results, kinetics follow a uniform pattern.

\subsection{Limitations of the study}

The most important limitation for us during the analysis of the results was the lack of criterion standard for diagnosing infection. Although we attempted to reduce the potential error in judgment by allocating patients into each group by 2 independent experts blinded for PCT kinetics in a post hoc fashion taking all clinical and microbiology data into account, one cannot exclude that mistakes may have still appeared in the process. Furthermore, the power analysis sample size could have been larger to detect a stronger signal, so a multicenter design would have been better. In addition, because of the exclusions, the sample size eventually included in the final analysis (141 patients) was substantially less than that calculated in the power analysis. It may also be important to note the uneven proportion of patients in the appropriate and inappropriate groups (75\% vs $25 \%$ ), a general limitation of every study in this field, which may also have affected our results. Finally, the clinical impact of our findings will have to be tested in a prospective randomized trial to see whether tailoring empirical antimicrobial therapy to PCT kinetics has any effect on outcome.

\section{Conclusion}

In this study, PCT kinetics within the first 24 hours after commencing empirical antimicrobial therapy showed a significant increase in patients in whom therapy proved to be inappropriate, whereas in the appropriate group, after a brief increase at 16 hours, there was a significant decrease by 24 hours. Applying this approach may be helpful in quickly tailoring antimicrobial therapy for the patient's specific needs. However, the clinical relevance of this "PCT kinetics-guided approach" should be confirmed in a prospective randomized fashion.

Supplementary data to this article can be found online at http://dx. doi.org/10.1016/j.jcrc.2016.04.007.

\section{Financial support and sponsorship}

None.

\section{Conflicts of interest}

JF and ZM have received lecture fees from ThermoFisher Scientific and BRAHMS GmbH. The other authors declare no conflicts of interest.

\section{Acknowledgment}

The authors express their special thanks to János Sándor and Ferenc Rárosi for their invaluable advice on statistics, Mrs Harriet Adamson for language editing, and the physicians and nurses in the ICU, without which this study could have not been completed.

\section{References}

[1] Dellinger RP, Levy MM, Rhodes A, Annane D, Gerlach H, Opal SM, et al. Surviving sepsis campaign: international guidelines for management of severe sepsis and septic shock: 2012. Crit Care Med 2013;41:580-637.

[2] Levy MM, Artigas A, Phillips GS, Rhodes A, Beale R, Osborn T, et al. Outcomes of the surviving sepsis campaign in intensive care units in the USA and Europe: a prospective cohort study. Lancet Infect Dis 2012;12:919-24.

[3] Kumar A, Roberts D, Wood KE, Light B, Parrillo JE, Sharma S, et al. Duration of hypotension before initiation of effective antimicrobial therapy is the critical determinant of survival in human septic shock. Crit Care Med 2006;34:1589-11596.

[4] Ferrer R, Martin-Loeches I, Phillips G, Osborn TM, Townsend S, Dellinger RP, et al. Empiric antibiotic treatment reduces mortality in severe sepsis and septic shock from the first hour: results from a guideline-based performance improvement program. Crit Care Med 2014;42:1749-55.

[5] Mettler J, Simcock M, Sendi P, Widmer AF, Bingisser R, Battegay M, et al. Empirical use of antibiotics and adjustment of empirical antibiotic therapies in a university hospital: a prospective observational study. BMC Infect Dis 2007;7:21. 
[6] Pierrakos C, Vincent JL. Sepsis biomarkers: a review. Crit Care 2010;1:R15

[7] Dandona P, Nix D, Wilson MF, Aljada A, Love J, Assicot M, et al. Procalcitonin increase after endotoxin injection in normal subjects. J Clin Endocrinol Metab 1994;79: 1605-8.

[8] Garnacho-Montero J, Huici-Moreno MJ, Gutierrez-Pizarraya A, Lopez I, MarquezVacaro JA, Macher H, et al. Prognostic and diagnostic value of eosinopenia, Creactive protein, procalcitonin, and circulating cell-free DNA in critically ill patients admitted with suspicion of sepsis. Crit Care 2014;18:R116.

[9] Muller B, Becker KL, Schachinger H, Rickenbacher PR, Huber PR, Zimmerli W, et al. Calcitonin precursors are reliable markers of sepsis in a medical intensive care unit. Crit Care Med 2000;28:977-83.

[10] Christ-Crain M, Jaccard-Stolz D, Bingisser R, Gencay MM, Huber PR, Tamm M, et al. Effect of procalcitonin-guided treatment on antibiotic use and outcome in lower respiratory tract infections: cluster-randomised, single-blinded intervention trial. Lancet 2004;363:600-7.

[11] Bouadma L, Luyt CE, Tubach F, Cracco C, Alvarez A, Schwebel C, et al. Use of procalcitonin to reduce patients' exposure to antibiotics in intensive care units (PRORATA trial): a multicentre randomised controlled trial. Lancet 2010;375:463-74.

[12] Dahaba AA, Elawady GA, Rehak PH, List WF. Procalcitonin and proinflammatory cytokine clearance during continuous venovenous haemofiltration in septic patients. Anaesth Intensive Care 2002;30:269-74.

[13] Annborn M, Dankiewicz J, Erlinge D, Hertel S, Rundgren M, Smith JG, et al. Procalcitonin after cardiac arrest - an indicator of severity of illness, ischemia-reperfusion injury and outcome. Resuscitation 2013;84:782-7.

[14] Calandra T, Cohen J. International sepsis forum definition of infection in the ICUCC. The international sepsis forum consensus conference on definitions of infection in the intensive care unit. Crit Care Med 2005;33:1538-48.

[15] Charles PE, Tinel C, Barbar S, Aho S, Prin S, Doise JM, et al. Procalcitonin kinetics within the first days of sepsis: relationship with the appropriateness of antibiotic therapy and the outcome. Crit Care 2009;13:R38.

[16] Clec'h C, Fosse JP, Karoubi P, Vincent F, Chouahi I, Hamza L, et al. Differential diagnostic value of procalcitonin in surgical and medical patients with septic shock. Crit Care Med 2006;34:102-7.

[17] American Thoracic Society. Infectious Diseases Society of America. Guidelines for the management of adults with hospital-acquired, ventilator-associated, and healthcareassociated pneumonia. Am J Resp Crit Care Med 2005;171:388-416.

[18] Solomkin JS, Mazuski JE, Bradley JS, Rodvold KA, Goldstein EJ, Baron EJ, et al. Diagnosis and management of complicated intra-abdominal infection in adults and children: guidelines by the Surgical Infection Society and the Infectious Diseases Society of America. Clin Infect Dis 2010;50:133-64.

[19] Mermel LA, Allon M, Bouza E, Craven DE, Flynn P, O'Grady NP, et al. Clinical practice guidelines for the diagnosis and management of intravascular catheter-related infection: 2009 update by the Infectious Diseases Society of America. Clin Infect Dis 2009;49:1-45.
[20] Trasy D, Nemeth M, Osztroluczki A, Tanczos K, Oveges N, Hankovszky P, et al. Early procalcitonin kinetics may indicate effective empirical antibiotic therapy within hours after starting treatment (a pilot study). Intensive Care Med 2013;39(Suppl. 2):78.

[21] Bone RC, Fisher Jr CJ, Clemmer TP, Slotman GJ, Metz CA, Balk RA. Sepsis syndrome: a valid clinical entity. Methylprednisolone severe sepsis study group. Crit Care Med 1989;17:389-93.

[22] American College of Chest Physicians. Society of Critical Care Medicine Consensus Conference: definitions for sepsis and organ failure and guidelines for the use of innovative therapies in sepsis. Crit Care Med 1992;20:864-74.

[23] Galicier C, Richet H. A prospective study of postoperative fever in a general surgery department. Infect Control 1985;6:487-90.

[24] Vincent JL. Dear SIRS, I'm sorry to say that I don't like you. Crit Care Med 1997;25: $372-4$.

[25] Ohl CA, Luther VP. Antimicrobial stewardship for inpatient facilities. J Hosp Med 2011;6(Suppl. 1):S4-15.

[26] Simon L, Gauvin F, Amre DK, Saint-Louis P, Lacroix J. Serum procalcitonin and Creactive protein levels as markers of bacterial infection: a systematic review and meta-analysis. Clin Infect Dis 2004;39:206-17.

[27] Kumar A, Ellis P, Arabi Y, Roberts D, Light B, Parrillo JE, et al. Initiation of inappropriate antimicrobial therapy results in a fivefold reduction of survival in human septic shock. Chest 2009;136:1237-48.

[28] Becker KL, Nylen ES, White JC, Muller B, Snider Jr RH. Clinical review 167: procalcitonin and the calcitonin gene family of peptides in inflammation, infection, and sepsis: a journey from calcitonin back to its precursors. J Clin Endocrinol Metab 2004;89:1512-25.

[29] Monneret G, Venet F, Pachot A, Lepape A. Monitoring immune dysfunctions in the septic patient: a new skin for the old ceremony. Mol Med 2008;14:64-78.

[30] Sfeir T, Saha DC, Astiz M, Rackow EC. Role of interleukin-10 in monocyte hyporesponsiveness associated with septic shock. Crit Care Med 2001;29:129-33.

[31] Heumann D, Glauser MP, Calandra T. Monocyte deactivation in septic shock. Curr Opin Infect Dis 1998;11:279-83.

[32] Nakamura A, Wada H, Ikejiri M, Hatada T, Sakurai H, Matsushima Y, et al. Efficacy of procalcitonin in the early diagnosis of bacterial infections in a critical care unit. Shock 2009;31:586-91.

[33] Uzzan B, Cohen R, Nicolas P, Cucherat M, Perret GY. Procalcitonin as a diagnostic test for sepsis in critically ill adults and after surgery or trauma: a systematic review and meta-analysis. Crit Care Med 2006;34:1996-2003.

[34] Kaczmarek A, Vandenabeele P, Krysko DV. Necroptosis: the release of damageassociated molecular patterns and its physiological relevance. Immunity 2013;38: 209-23.

[35] Meisner M, Tschaikowsky K, Hautzler A, Schick C, Schüttler J. Postoperative plasma concentrations of prokalcitonin after different types of surgery. Intensive Care Med $1998 ; 24: 680-4$ 